Journal of Classification 33:523 (2016)

DOI: 10.1007/s00357-016-9219-1

\title{
REVIEWERS
}

\section{Guest Reviewers, Journal of Classification Volume 33, 2016}

In addition to the members of the Editorial Board, the individuals listed below refereed manuscripts that were submitted to the journal. Their assistance is gratefully acknowledged.

$\begin{array}{ll}\text { Bart Baesens } & \text { Edgar Merkle } \\ \text { Norma Bargary } & \text { Geoff McLachlan } \\ \text { Laine Bradshaw } & \text { Paul McNicholas } \\ \text { Tim Brennan } & \text { Boris Mirkin } \\ \text { James Brown } & \text { Brendan Murphy } \\ \text { Ryan Browne } & \text { Fionn Murtagh } \\ \text { Michael Brusco } & \text { Alicia Nieto-Reyes } \\ \text { Frank Busing } & \text { Hans-Peter Piepho } \\ \text { Paola Cerchiello } & \text { Jeffrey Rouder } \\ \text { Steven Culpepper } & \text { Florentin Smarandache } \\ \text { Jimmy de la Torre } & \text { Stanley L. Sclove } \\ \text { Pierpaolo D'Urso } & \text { Emilie Shireman } \\ \text { Holmes Finch } & \text { Jordan Stevens } \\ \text { Stephen France } & \text { Clintin Stober } \\ \text { Pasi Franti } & \text { Zdenek Sulc } \\ \text { Kathleen Gates } & \text { Yoshio Takane } \\ \text { Gregory Gay } & \text { Thaddeus Tarpey } \\ \text { John Gower } & \text { Valentin Todorov } \\ \text { Kiero Guerra } & \text { ShengLi Tzeng } \\ \text { Jeffrey Harring } & \text { Iven Van Mechelen } \\ \text { Christian Hennig } & \text { Marika Vezzoli } \\ \text { Michaela Hoffman } & \text { Sunny Wang } \\ \text { Xiaopeng Hua } & \text { Matthijs J Warrens } \\ \text { Henk Kiers } & \text { Philip Wood } \\ \text { Hans Friedrich Köhn } & \text { Yoshio Takane } \\ \text { Vipin Kumar } & \\ \text { Herbert Lee } & \\ \text { Rosaria Lombardo } & \\ \text { Ann Maharaj } & \\ \text { Elizabeth A Maharaj } & \\ \text { Ranjan Maitra } & \\ \text { Matthieu Marbac } & \end{array}$

